\title{
THE POINTWISE CHARACTERIZATION OF DERIVATIVES OF INTEGRALS
}

\author{
D. N. SARKHEL ${ }^{1}$
}

\begin{abstract}
Let $\sigma$ denote the indefinite integral of a summable function $f$ on $R_{n}$. We give necessary and sufficient conditions for $\sigma$ to possess a strong, or ordinary, or general derivative, equal to $f(x)$ at a point $x$ of approximate continuity of $f$. Munroe [3] states that when $f$ is a bounded function then $f(x)$ is the strong derivative of $\sigma$ at $x$ if and only if $x$ is a point of approximate continuity of $f$. We point out an error in the proof of the 'only if' part of this result and show by example that this part of Munroe's result is in fact false.
\end{abstract}

We begin with what forms an important part of this note, a remark on the following theorem of Munroe [3, Theorem 42.4]:

If $f$ is bounded and integrable over $R_{n}$ and if $\sigma=\int f$, then a necessary and sufficient condition that $\operatorname{D\sigma }(x)=f(x)$ is that $x$ be a point of approximate continuity for $f$.

Here $R_{n}$ denotes the Euclidean $n$-space, the integral is in Lebesgue sense, the term integrable means summable, $\sigma=\int f$ denotes the indefinite integral defined by $\sigma(B)=\int_{B} f$ for measurable sets $B \subset R_{n}$, and $D \sigma(x)$ denotes the strong derivative of $\sigma$ at the point $x \in R_{n}$. The sufficiency of the condition is well known (cf. [4, Theorem 10.7]); what appears to be new is its necessity. In the course of his proof of necessity [3, p. 293], Munroe asserts that

$$
\frac{\sigma(J)}{\mu(J)} \geqslant \frac{\sigma(J \cap E)}{\mu(J)} \geqslant \varepsilon \cdot \frac{\mu(J \cap E)}{\mu(J)},
$$

where $\mu$ denotes the Lebesgue measure function, $J$ denotes any interval containing the point $x, E=\{y \mid f(y) \geqslant \varepsilon\}, \varepsilon>0$, and it is assumed that $f(x)=0$. Unfortunately, in (*) the first inequality is invalid. In fact the condition in Theorem 42.4 is not really necessary. The following is a counterexample. Let $A$ be a bounded measurable subset of $R_{n}$ having unique (outer) density $\alpha, 0<\alpha<1$, at a point $c$. In $R_{1}$, for instance, the set

$$
A_{0}=\bigcup_{k=1}^{\infty}\left(\left(a_{k}, b_{k}\right) \cup\left(-b_{k},-a_{k}\right)\right),
$$

Received by the editors August 26, 1975 and, in revised form, March 24, 1976.

AMS (MOS) subject classifications (1970). Primary 28A15.

Key words and phrases. Euclidean $n$-space, strong derivative, general derivative, ordinary derivative, density, approximate continuity, summable.

${ }^{1}$ The author thanks the referee for suggesting some improvements of the text. 
where

$$
a_{k}=\frac{1}{2 k}+\frac{1}{2 k+2}+\frac{1}{2^{k+2}}, \quad b_{k}=\frac{1}{k}+\frac{1}{2^{k+1}},
$$

is bounded and measurable, and has unique density $\frac{1}{2}$ at the origin. Let $G$ be a bounded open set containing the set $A \cup\{c\}$ and let $f: R_{n} \rightarrow R_{1}$ be defined by

$$
f(x)= \begin{cases}1-\alpha & \text { if } x \in A \backslash\{c\}, \\ -\alpha & \text { if } x \in G \backslash(A \cup\{c\}), \\ 0 & \text { otherwise. }\end{cases}
$$

Then $f$ is bounded and summable over $R_{n}$. If $\left\{J_{k}\right\}$ is any sequence of intervals in $R_{n}$ tending to $c$ such that $J_{k} \subset G$ for each $k$, then

$$
\begin{aligned}
\frac{\sigma\left(J_{k}\right)}{\mu\left(J_{k}\right)} & =\frac{(1-\alpha) \cdot \mu\left(J_{k} \cap A\right)-\alpha \cdot \mu\left(J_{k} \backslash A\right)}{\mu\left(J_{k}\right)} \\
& =\frac{\mu\left(J_{k} \cap A\right)}{\mu\left(J_{k}\right)}-\alpha \rightarrow 0 .
\end{aligned}
$$

Thus $D \sigma(c)=0=f(c)$. Nevertheless, $f$ is not approximately continuous at $c$. We note that if $J \subset G$ is any interval containing $c$, and $E=\{x \mid f(x) \geqslant \varepsilon\}$, $0<\varepsilon<1-\alpha$, then $E=A \backslash\{c\}$ and hence

$$
\sigma(J)=\sigma(J \cap E)-\alpha \cdot \mu(J \backslash E)<\sigma(J \cap E) \text {; }
$$

therefore the first inequality in $(*)$ fails in this case.

Next, to quote Munroe [3, p. 293 infra], "there seems to be no respectable sufficient condition that $D \sigma(x)=f(x)$ in the case of unbounded $f$ ". It is worth mentioning in this connection that a sufficient condition to ensure $D \sigma(x)=f(x)$ a.e. has been obtained in [2]. On the other hand, for $n \geqslant 2$ there exist unbounded functions $f$ such that the relation $D \sigma(x)=f(x)$ does not hold at any point of approximate continuity of $f$ (cf. [1, p. 256]). In any case however, the general derivative as well as the ordinary derivative of $\sigma$ exists and equals $f$ a.e. (cf. [4, Theorem 6.3]).

The strong derivative, the ordinary derivative and the general derivative of a function of sets in $R_{n}$ are defined (cf. [4, p. 106]) respectively in terms of "sequences of arbitrary intervals", "regular sequences of intervals" and "regular sequences of closed sets". Correspondingly (using these three derivatives of the measure function of a subset $A$ of $R_{n}$ ) three kinds of densities for $A$ can be defined: the strong density, the ordinary density and the general density. In particular strong density and outer density (cf. [4, p. 128]) are synonymous.

An a.e. finite point function $f$ on $R_{n}$ is said to be approximately continuous at a point $c$ if and only if for every real number $\varepsilon>0$ the set $\{x|| f(x)-$ $f(c) \mid \geqslant \varepsilon\}$ has strong density zero at $c$. In this definition, replacing "strong density" first by ordinary density and second by general density we get two other kinds of approximate continuity. These three kinds of approximate continuity will be referred to as strong approximate continuity, ordinary ap- 
proximate continuity and general approximate continuity, respectively. Obviously $f$ is measurable if and only if it is approximately continuous a.e. in all the three senses.

In the following theorem the terms "derivative", "density" and "approximate continuity" can be taken in either the strong, the ordinary, or the general sense, while $\Delta$ denotes derivation in the corresponding sense; $\Delta \tau(x)$ denotes the derivative of a set function $\tau$ at a point $x \in R_{n}$ and $\chi_{E}$ denotes the characteristic function of a subset $E$ of $R_{n}$.

THEOREM. Let $f$ be summable over $R_{n}$ and let $c$ be a point of approximate continuity of $f$. Let $\sigma=\int f, \sigma_{E}=\int \chi_{E} \cdot f$ for any fixed measurable subset $E$ of $R_{n}$, and $\mathcal{E}$ the family of measurable subsets $E$ which have density zero at $c$ and are such that $f$ is bounded on $R_{n} \backslash E$. Then in order that $\Delta \sigma(c)=f(c)$ it is necessary that for every $E \in \mathcal{E}$ we have $\Delta \sigma_{E}(c)=0$, and it is sufficient that for some $E \in \mathcal{E}$ we have $\Delta \sigma_{E}(c)=0$.

Proof. Fix $E \in \mathcal{E}$ and $\varepsilon>0$. Put

$$
h(x)=f(x)-f(c), \quad x \in R_{n} ; \quad A=\{x|| h(x) \mid \geqslant \varepsilon\} .
$$

Since $f$ is approximately continuous at $c$, the set $A$ has density zero at $c$. Now for any measurable set $B \subset R_{n}$ with $0<\mu(B)<\infty$, we have (writing $I(g)$ for $\int_{R_{n}} g$ )

$$
\begin{aligned}
\sigma(B)-f(c) & \cdot \mu(B)=I\left(\chi_{B} \cdot h\right) \\
= & I\left(\chi_{B \cap E} \cdot h\right)+I\left(\chi_{B \cap A \backslash E} \cdot h\right)+I\left(\chi_{B \backslash(A \cup E)} \cdot h\right) \\
= & \sigma_{E}(B)-f(c) \cdot \mu(E \cap B)+I\left(\chi_{B \cap A \backslash E} \cdot h\right)+I\left(\chi_{B \backslash(A \cup E)} \cdot h\right) .
\end{aligned}
$$

Transposing and using modulus inequalities, we get

$$
\begin{aligned}
& \left|\{\sigma(B)-f(c) \cdot \mu(B)\}-\sigma_{E}(B)\right| \\
& \quad \leqslant|f(c)| \cdot \mu(E \cap B)+I\left(\chi_{B \cap A \backslash E} \cdot|h|\right)+I\left(\chi_{B \backslash(A \cup E)} \cdot|h|\right) .
\end{aligned}
$$

Since $E \in \mathcal{E}$, there is a constant $M>0$ such that

$$
|h(x)| \leqslant M \text { for all } x \in R_{n} \backslash E \text {. }
$$

Also, by definition of the set $A$, we have

$$
|h(x)|<\varepsilon \quad \text { for all } x \in R_{n} \backslash A .
$$

Using these facts, and dividing through by $\mu(B)$, we get from (1)

$$
\begin{aligned}
\left|\left\{\frac{\sigma(B)}{\mu(B)}-f(c)\right\}-\frac{\sigma_{E}(B)}{\mu(B)}\right| \leqslant|f(c)| \\
\cdot \frac{\mu(E \cap B)}{\mu(B)}+M \cdot \frac{\mu(A \cap B)}{\mu(B)}+\varepsilon .
\end{aligned}
$$

On the other hand, for any of the three types of derivation $\Delta$, if $\left\{B_{k}\right\}$ is an appropriate sequence of sets tending to $c$, then

$$
\frac{\mu\left(E \cap B_{k}\right)}{\mu\left(B_{k}\right)} \rightarrow 0 \text { and } \frac{\mu\left(A \cap B_{k}\right)}{\mu\left(B_{k}\right)} \rightarrow 0 \text {. }
$$


The proof can now be completed by taking $B=B_{k}$ in (2) and using (3).

Note. The theorem evidently implies: $\Delta \sigma(c)=f(c)$ if and only if for some $E \in \mathcal{E}$ we have $\Delta \sigma_{E}(c)=0$.

ADDENDUM. After writing this paper the author came to know of a revised edition (M. E. Munroe, Measure and Integration, 1971, Addison-Wesley; referred to below by [3a]) of [3]. In Theorem 35.4 of [3a] Munroe states: If $f$ is nonnegative and integrable over $R_{n}$, if $\sigma=\int f$, and if $D \sigma(x)=f(x)$, then $x$ is a point of approximate continuity for $f$. His proof of this result is identical to that of the 'only if' part of Theorem 42.4 in [3]. We observe that in his proof Munroe actually considers instead of the function $f$ the function $f$ minus the constant $f(x)$, and hence his assertion (*) does not seem to be justifiable. In fact, even Theorem 35.4 in [3a], that is, the 'only if' part of Theorem 42.4 in [3] for nonnegative $f$, is false. Take for instance $f=\frac{1}{2} \chi_{\{c\}}+\chi_{A}$, where $c$ is a given point of $R_{n}$ and $A$ is a bounded measurable subset of $R_{n} \backslash\{c\}$ having strong density $\frac{1}{2}$ at $c$. Then $D \sigma(c)=f(c)$ but $f$ is not approximately continuous at $c$.

\section{REFERENCES}

1. H. Busemann and W. Feller, Zur Differentiation der Lebesgueschen Integrale, Fund. Math. 22 (1934), 226-256.

2. B. Jessen, J. Marcinkiewicz and A. Zygmund, Note on the differentiability of multiple integrals, Fund. Math. 25 (1935),217-234.

3. M. E. Munroe, Introduction to measure and integration, Addison-Wesley, Reading, Mass., 1953; 1959. MR 14, 734.

4. S. Saks, Theory of the integral, 2nd rev. ed., Monografie Mat., vol. VII, PWN, Warsaw, 1937; reprint, Dover, New York, 1964. MR 29 \#4850.

Department of Mathematics, University of Kalyani, Nadia, West Bengal, India 